\title{
Abstract numerical discrimination learning in rats
}

\author{
Tohru Taniuchi $^{1} \cdot$ Junko Sugihara $^{2} \cdot$ Mariko Wakashima $^{2} \cdot$ Makiko Kamijo $^{1}$
}

Published online: 28 January 2016

(C) Psychonomic Society, Inc. 2016

\begin{abstract}
In this study, we examined rats' discrimination learning of the numerical ordering positions of objects. In Experiments 1 and 2, five out of seven rats successfully learned to respond to the third of six identical objects in a row and showed reliable transfer of this discrimination to novel stimuli after being trained with three different training stimuli. In Experiment 3, the three rats from Experiment 2 continued to be trained to respond to the third object in an object array, which included an odd object that needed to be excluded when identifying the target third object. All three rats acquired this selective-counting task of specific stimuli, and two rats showed reliable transfer of this selective-counting performance to test sets of novel stimuli. In Experiment 4, the three rats from Experiment 3 quickly learned to respond to the third stimulus in object rows consisting of either six identical or six different objects. These results offer strong evidence for abstract numerical discrimination learning in rats.
\end{abstract}

Keywords Rat $\cdot$ Selective counting $\cdot$ Absolute ordering · Numerical processing $\cdot$ Discrimination

Electronic supplementary material The online version of this article (doi:10.3758/s13420-016-0209-2) contains supplementary material, which is available to authorized users.

Tohru Taniuchi

tohruta@staff.kanazawa-u.ac.jp

1 Graduate School of Socio-Environmental Studies, Kanazawa University, Kakuma, 920-1192 Kanazawa, Ishikawa, Japan

2 Faculty of Letters, Kanazawa University, Kakuma, Kanazawa, Ishikawa, Japan
A long-standing topic in comparative psychology is whether nonhuman animals can learn the abstract concept of number. Numerical competence allows animals to comprehend the numerical aspects of a variety of aggregations that differ in specific physical features. Using the abstract concept of number, we can apply common calculation rules to a wide variety of stimuli.

The abstract concept of number has been divided into two components: concepts of abstract numerosity and of the order of numerosities (Brannon \& Roitman, 2003; Gelman \& Gallistel, 1978; Neider, 2005). Abstract numerosity is suggested by responding to the numerosities of stimuli, regardless of their physical properties, such as size, color, shape, and so on. The concept of ordering relationships among these abstract numerosities enables animals to recognize, for example, that "fourness is greater than threeness and less than fiveness."

Various animal species have proven their ability to learn abstract numerosities. For example, a chimpanzee (Matsuzawa, 1985) and pigeons (Xia, Emmerton, Siemann, \& Delius, 2001; Xia, Siemann, \& Delius, 2000) have shown that they could respond correctly to the number of visual stimuli using arbitrary symbols. Rhesus monkeys (Brannon \& Terrace, 1998, 2000) and capuchin monkeys (Judge, Evans, \& Vyas, 2005) can respond to the ordinal relationship of a number of different figures on a computer monitor correctly. A male African gray parrot could give a vocal answer to the number of specific objects or the sum of two numbers (Pepperberg, 1994, 2012). More recently, precise discrimination of small numerosities or rough discrimination of large numerosities has been reported for various species - for example, elephants (Perdue, Talbot, Stone, \& Beran, 2012), infant chicks (Rugani, Regolin, \& Vallortigara, 2008), and guppies (Miletto Petrazzini, Agrillo, Izard, \& Bisazza, 2015).

A number of studies have also been conducted on numerical competence in rats. For example, it has been reported that rats can discriminate the number of reinforced runs in a 
straight runway (Capaldi \& Miller, 1988), the number of touches to their body (Davis, MacKenzie, \& Morrison, 1989), the number of auditory tones (Breukelaar \& Dalrymple-Alford, 1998; Davis \& Albert, 1986), and the number of lined tunnels in an open field (Davis \& Bradford, 1986; Suzuki \& Kobayashi, 2000). However, excluding an exceptional study, which tested the transfer of numerical discrimination of auditory stimuli to visual stimuli and showed no sign of transfer of learning (Davis \& Albert, 1987), the abstractness of numerical concepts in rats has not been elucidated because the clear transfer of counting behavior to novel stimuli has not yet been documented in rats.

Recently, Kamijo and Taniuchi (2015) found that rats could successfully learn to respond to the third stimulus in an object row consisting of identical objects (see Figs. 1 and 2 below). Four to six identical objects were placed in front of ten goal boxes, and the spatial position of the target stimulus was changed trial by trial to prevent it from serving as an effective discriminative cue. However, despite their successful acquisition of this task, they failed to show clear transfer of discrimination to novel test stimuli, even after acquisition with six different training stimuli. The simplest explanation of the results is the absence of abstract numerical competence in rats. That is, rats might learn a certain kind of stimulus-specific cue for each object. Nonhuman animals frequently learn and use a stimulus-specific cue-for example, the specific configuration of multiple stimuli- to solve a relational conceptlearning task, particularly when a small number of training stimuli are used (e.g., Wright \& Katz, 2006).

However, we need to examine several other possibilities before concluding that rats lack an abstract numerical competence. First, it is known that the novelty of test stimuli in a matching-to-sample task interferes with test performance, provoking exploration of the novel stimuli in monkeys (D'Amato, Salmon, \& Colombo, 1985). It has also been shown that rats exhibit not only an exploration tendency (e.g., Ennaceur, Michalikova, \& Chazot, 2009), but also neophobia, an avoidance tendency (Wallace \& Barnett, 1990), toward novel test objects. In Kamijo and Taniuchi (2015), rats showed a significant avoidance tendency toward novel test stimuli and frequently responded to a goal box with no test object in front of it. Therefore, in Kamijo and Taniuchi, test performance could have been affected by neophobia toward the novel test stimuli. In the present study, rats were habituated to the test stimuli before the stimuli were used in test phases, increasing familiarity with the test stimuli and reducing a possible exploration tendency or neophobia toward them, which might interfere with test performance.

A second possible problem in Kamijo and Taniuchi (2015) is related to the procedure, in which food rewards were placed in all goal boxes in order to control for a possible olfactory cue, whereas the small one-way doors immediately in front of the food cups in incorrect goal boxes were locked so that rats
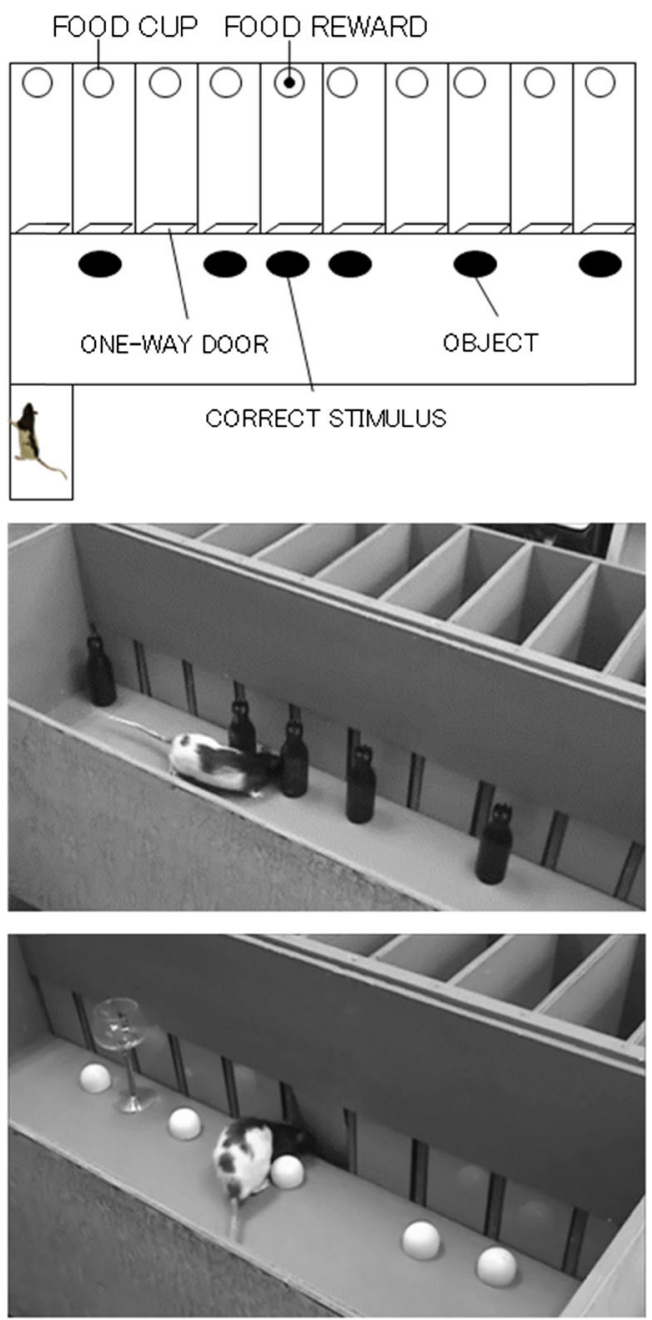

Fig. 1 Diagram of the apparatus (top) and photos of performance during the acquisition task of Experiment 1 (middle) and the selective-counting task of Experiment 3 (bottom)

could only access food rewards in correct goal boxes. Informally, the experimenters observed that rats sometimes

Trial 1

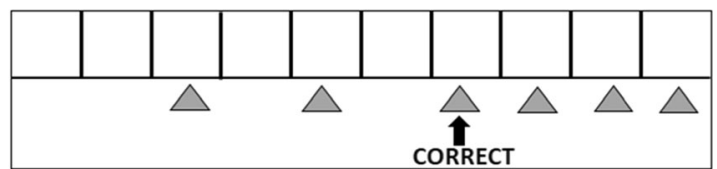

Trial 2

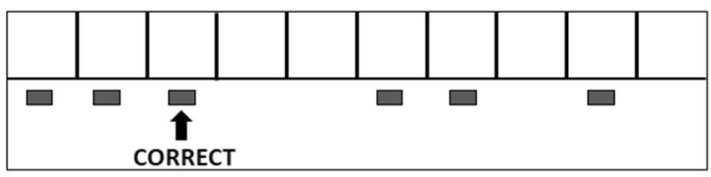

Trial 3

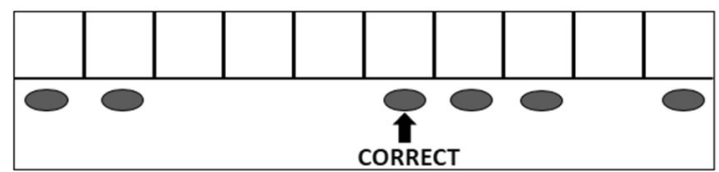

Fig. 2 Examples of object arrangements for Phase 5 trials in Experiment 1, in which three types of objects, A, B, and C, were used for training. Only a single type of object was used per trial, and the object types were changed every three trials 
persisted in trying to open the small one-way door in incorrect goal boxes to get at the food reward behind it, possibly due to an olfactory cue from the food reward. Although this procedure may have been an appropriate way to control for a possible odor cue from food rewards, a persistent, irrelevant behavior in an incorrect goal box might have interfered with acquisition of numerical discrimination learning. That is, when rats experience a reinforcement event (either permitting or denying access to food rewards), they need to associate the reinforcement event with the stimulus they responded to. Given that it was impossible for a rat inside a goal box to perceive the object outside the box, rats needed to associate the reinforcement event in short-term memory with information about the discriminative stimulus. Thus, persistent emission of irrelevant responses possibly interfered with the rats forming associations between discriminative responses and reward events in short-term memory. To avoid interfering with association formation, food rewards were only put in the correct goal box during the later training phases. The influence of an olfactory cue from the food reward was controlled on test trials by nondifferential reinforcement- that is, placing food rewards in incorrect goal boxes as well as the correct goal box.

The goal of the present study was to reexamine whether rats can learn an absolute ordering position of the stimuli in an object row at an abstract level with some methodological modifications of Kamijo and Taniuchi (2015). Rats were trained to respond to the third object in an object row consisting of identical objects. After acquisition of this discrimination, several tests were conducted to assess whether rats could learn to transfer this discrimination to novel objects and varying numbers of objects, in order to elucidate whether they could learn an abstract numerical property of discriminative stimuli.

\section{Experiment 1}

In Experiment 1, we examined whether rats could learn a specific ordering position in an object row and transfer this discriminative behavior to novel stimuli. Four rats were trained to respond to the third of six identical objects arranged in a row. The spatial position of the correct stimulus was varied trial by trial to prevent spatial location from being an effective discriminative stimulus.

Unlike Kamijo and Taniuchi (2015), who placed food rewards in all goal boxes, food rewards were put only in the correct goal boxes behind the correct stimulus during training, to avoid the possibly disruptive behavior of trying to get food rewards in an incorrect box. Thus, the odor cue might have been available as an effective discriminative cue during acquisition training. After learning to respond reliably with three different training stimuli, probe test trials with novel objects were inserted into the training trials. On a probe test trial, food rewards were put in goal boxes behind the second, third, and fourth stimuli in an object row, to evaluate the possible effect of an olfactory cue. This selective nondifferential reinforcement probe test is a variant of a nondifferential reinforcement probe test in which any choice of response is reinforced (e.g., Castro, Lazareva, Vecera, \& Wasserman, 2010). We reinforced only the second, third, and fourth objects, not all objects, because our preliminary investigation showed that complete nondifferential reinforcement or extinction treatment to the test stimuli disrupted rats' baseline performance completely. If rats learned to respond to the odor cue from food rewards, response rates should be equal across the second, third, and fourth objects, because these objects were reinforced equally. By contrast, if a rat transferred its discriminative response to the third stimulus to novel test stimuli, we would expect response rates to the third object to be significantly better than chance, even on probe test trials.

\section{Method}

Subjects The subjects were four experimentally naive, male Long-Evans rats, approximately 60 days old at the beginning of the experiment. Rats were given $14 \mathrm{~g}$ of food daily, except for the experimental rewards. These rats were acquired from Kiwa Laboratory Animals Company, Ltd., and were cared for and used according to guidelines approved by Kanazawa University Animal Experimentation Regulations.

Apparatus Figure 1 shows a schema of the apparatus and photos of performance in Experiments 1 and 3. Ten goal boxes, $35 \mathrm{~cm}$ long, $10 \mathrm{~cm}$ wide, and $40 \mathrm{~cm}$ high, were lined up next to each other in the apparatus. Each goal box had a oneway swing door at its entrance. A food cup was placed at the end of the goal box, and two 0.045 -g food pellets were placed in the cups as a food reward. The apparatus was painted flat gray, and the one-way doors were made of gray PVC board. The objects used in the initial training and transfer tests were glass bottles, metallic 350-ml cans, hemispherical capsules, and rhinoceros beetle models. The assignment of these objects to items $\mathrm{A}, \mathrm{B}, \mathrm{C}$, and $\mathrm{D}$ was counterbalanced among subjects. A glass fish tank, $60 \mathrm{~cm}$ long, $30 \mathrm{~cm}$ wide, and $36 \mathrm{~cm}$ high, was used for exploration of the test objects in advance of testing.

Procedure During the first 7 days, the rats were handled for $3 \mathrm{~min}$, and ten food pellets were placed in their home cages for familiarization. Exploration of the apparatus was allowed on Days 8 and 9 for 20 min individually. All doors were open, and rats could eat the food pellets scattered over the apparatus floor and food cups. From Day 10, the rats were shaped to open the one-way doors. A rat was put in the start box, and then the guillotine door was opened about $3 \mathrm{~s}$ later. Nine of the ten doors were blocked, and rats could only enter the single 
open goal box. Rats were eventually trained to enter a completely closed door.

After the completion of shaping, Phase 1 of acquisition training with stimulus A commenced. Figure 2 shows how the objects were placed for a trial. Six objects were arranged in random order in front of the ten goal boxes, and the rats were trained to choose the goal box with any object A in front. Correct responses were rewarded by two food pellets. For an incorrect response, rats were confined in the goal box with no reward for $10 \mathrm{~s}$. The intertrial interval was 4-8 $\mathrm{min}$. When rats had attained the learning criterion of $70 \%$ correct in a daily 24-trial session, Phase 2 training was started. In Phase 2, responses to the second, third, and fourth objects, counting from the left in the object row, were reinforced. The other aspects of the procedure and the learning criterion were identical to Phase 1. In Phase 3, the correct response was restricted to the third object. Possible correct (third) goal boxes for the arrangement of the six objects were the third, fourth, fifth, sixth, or seventh in the row of ten boxes. The frequencies of the five goal boxes as the correct box were made equal and counterbalanced over five trials. The learning criterion for Phase 3 was $70 \%$ correct in a daily 24-trial session, or $50 \%$ correct for four successive sessions. In Phase 4, object B was added to the training, and objects $\mathrm{A}$ and $\mathrm{B}$ were each used for 12 daily trials in a daily 24-trial session. The order of trials with objects A or B was randomized for every two trials. The learning criterion was now $50 \%$ correct for both A and B individually, and $80 \%$ correct in total for a session or $70 \%$ correct for three successive sessions. In Phase 5, object $\mathrm{C}$ was added to the training, and eight trials were given for each object. The learning criterion was adjusted to $50 \%$ correct for each of the three objects and $80 \%$ correct in total for four successive sessions. The order of trials with these three objects was randomized for every three trials. On the day a rat attained the learning criterion for Phase 5, exploration of the test stimuli was provided after training, to habituate the rats to the new stimuli. Four identical test objects D were placed in a glass fish tank, set in an identical experimental room, and each rat was individually allowed to explore them freely for $20 \mathrm{~min}$.

After the completion of Phase 5, probe tests were conducted for 6 days. In this phase, 21 training trials with object A, B, or $\mathrm{C}$ and three probe trials with novel object $\mathrm{D}$ were conducted in each daily 24-trial session. The procedure for the probe test was identical to that of Phase 5, except for the following two points. A probe trial with $\mathrm{D}$ was inserted after every seventh training trial, and responses to the second, third, and fourth objects from the left in the object row were reinforced nondifferentially for test stimulus $\mathrm{D}$. The reason for reinforcing these three objects was as follows: By reinforcing the second, third, and fourth objects, we could evaluate two possible nontransfer effects - that is, utilization of an olfactory cue from food rewards and training effects during the test period. That is, if rats responded to olfactory cues from the food rewards, they should respond to the second, third, and fourth test objects equally. Similarly, if they learned to respond to novel test stimulus D on the basis of reinforced experience on the test trials, rats should also respond to these three objects equally. Conversely, if a rat responded to the third object more frequently than to either the second or fourth object, this could be attributed to a transfer effect from acquisition training.

Following the probe test, rats were trained with objects A, B, C, and D in Training Phase 6 until they had attained the learning criterion of $50 \%$ correct for each of the three objects and $70 \%$ correct in total in four successive sessions. After acquisition with four different objects, the total number of objects presented in a trial varied randomly among four, five, or six objects across trials, to evaluate the possibility that rats counted the objects from the right end of an object row or used the relative position in an object row to identify a target object. That is, if rats counted objects from the far right end of the row, it was expected that their performance would deteriorate when the total number of objects was changed from six to four or five, because the ordinal position of the target object from the right end varied, depending on the total number of objects in a row. By contrast, if rats counted objects from the left end, the total number of objects would not influence rats' performance, because the ordinal position of the target object remained the third place, regardless of the total number of objects. Similarly, if the total number of objects was six, the correct third stimulus was always located just to the left of the point dividing the row of six objects in half. However, the correct stimulus was placed just to the right of the halfway mark for a four-object row, or at the center of the five-object row. Therefore, if rats learned the relative position of the correct stimulus in the object row, their performance would deteriorate when the total number of objects was changed from six to four or five. Only responses to the third object were reinforced. For the four- and five-object conditions, we maintained the same possible positions for the correct goal box as in the six-object condition, thereby excluding the first, second, eighth, ninth, and tenth boxes as correct goal boxes. The order of these three different conditions was randomized for every three trials, and this test was conducted for two sessions.

\section{Results}

Figure 3 shows the percentages of correct responses during acquisition training. Vertical lines in the figure represent shifts of training phase. Rats 1 and 2 were excluded from training because their performance did not improve and remained at a low level. In contrast, Rats 3 and 4 attained the learning criterion of Phase 5 acquisition training with three different stimuli, A, B, and C.

We can assume several different chance levels for the acquisition training phases. The first would be the "objects" model, with a chance level of $16.67 \%$, which is the expected 


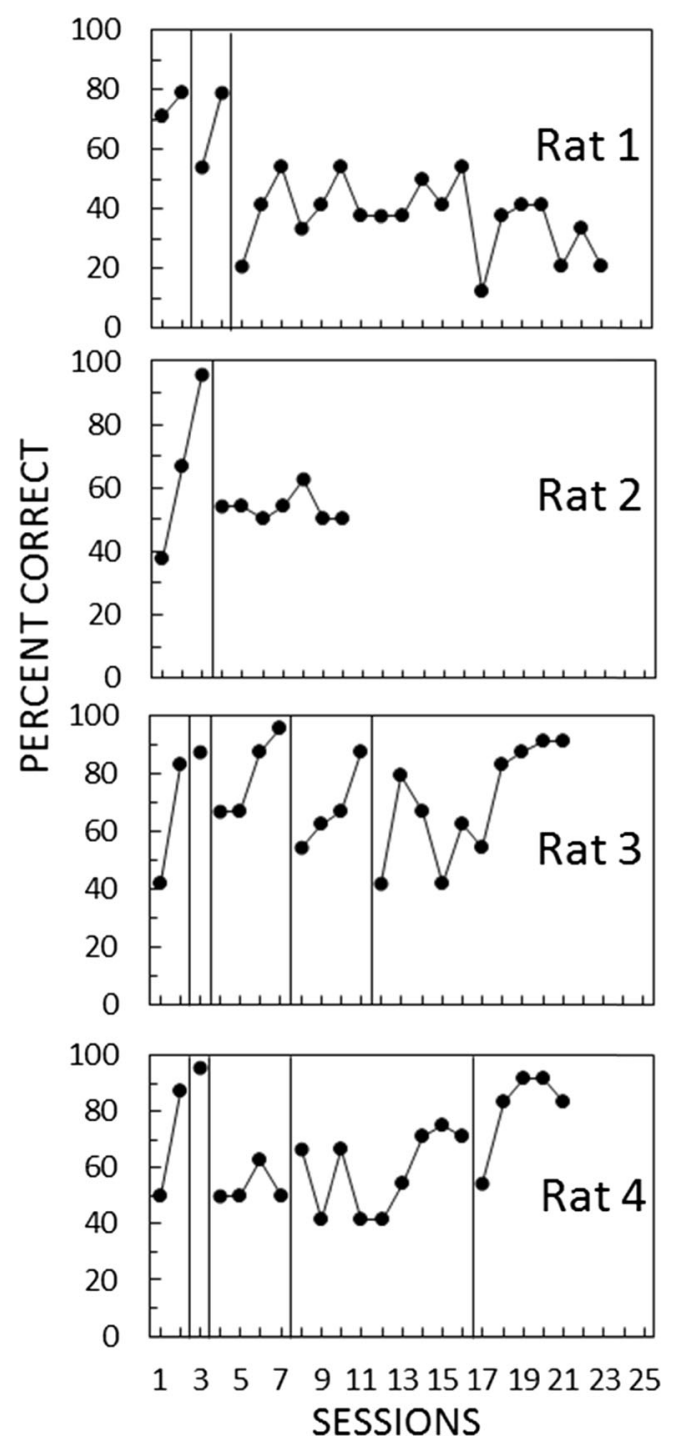

Fig. 3 Percentages of correct responses during acquisition training for Experiment 1 . The solid vertical lines represent the beginning of each new training phase

value when rats responded randomly to the six presented objects. The second would be the "possible goals" model, set at $20 \%$, which would be expected if rats respond randomly to the five possible goal boxes - that is, the third to the seventh boxes, because the target third object could not be placed in front of the first, second, eighth, ninth, or tenth goal box in a row of six objects. The third chance level, according to the "objects and goals" model, would be set at $35.93 \%$ correct responses, if rats responded randomly to objects in front of the five possible goal boxes. That is, there were 35 patterns of object arrangements in which the target third object was set in front of Goal 3. In these 35 arrangements, 95 objects in total could be set in front of possible Goals 3-7. Therefore, in the case of the third box being correct, the chance level would be $36.84 \%(35 / 95)$, with rats responding to the objects in front of the possible goals randomly. Similarly, the chance level would be $31.58 \%(60 / 190), 34.45 \%$ (60/174), $33.90 \%$ (40/118), or $42.85 \%(15 / 35)$ in each case, if the target third object was set in front of Goals 4, 5, 6, and 7, respectively. Since we used these possible goals with equal frequencies, the total chance level of the "objects and goals" model could be set at $35.93 \%$, an average of the chance levels for Goals 3-7. Thus, we assumed that $35.93 \%$ would be the most conservative chance level to evaluate a rat's performance in acquisition training. The performance of Rats 3 and 4 on the last four sessions of Phase 5 was significantly higher than the most conservative chance level (one-tailed binomial test, $p \mathrm{~s}<.01$ ).

Figure 4 shows the results of the probe test with novel stimulus $\mathrm{D}$. The rats made some errors by choosing the second and fourth objects, but they never responded to objects in more remote ordinal positions or goals with no object. We reinforced responses to the second, third, and fourth objects in an object row to control for olfactory cues from the food rewards or learning effects during the test trials. Thus, the chance level was $1 / 3=33.33 \%$ for these two possible nonnumerical choices on test trials (models of "olfactory cue" and "reinforced experience"). Rats 3 and 4 both made correct responses at a significantly higher than chance level when compared to the $33.33 \%$ chance level for olfactory cues from food rewards or from learning effects (one-tailed binomial test, $p \mathrm{~s}<.01$ ), but also when compared to the more conservative "objects and goals" model chance level of $35.95 \%$ (onetailed binomial test, $p \mathrm{~s}<.01$ ).

However, if rats combined olfactory cues during the test trials with possibly correct goal boxes - that is, if rats responded randomly to a goal box from Boxes 3-7 with an olfactory cue from a food reward - the chance level for correct response would be $43.00 \%$ ("olfactory cues and possible goals" model). That is, there were 35 patterns of object arrangement in which the target third object was set in front of the possible Goal 3. In these 35 patterns, the second object was never placed in front of possible Goals $3-7$, and the fourth object could be set in front of these possible goals in 34 patterns of object arrangements. Therefore, if rats responded to the olfactory cue presented in the possible Goals 3-7, the chance level would be $50.72 \%(35 / 69)$. There were 60 patterns of object arrangement in which the target stimulus was set in front of Goal 4. In these 60 patterns, the second, third, and fourth objects could be set in front of the possible goals in 40, 60, and 57 patterns, respectively. Therefore, the chance level of the "olfactory cues and possible goals" model would be $38.22 \%(60 / 157)$ when the third object was placed in front of Goal 4. Similarly, the chance levels of the "olfactory and possible goals" model would be $36.59 \%$ (60/164), $37.74 \%$ $(40 / 106)$, and $51.72 \%(15 / 29)$ when the third object was set in front of possible Goals 5, 6, and 7, respectively. Since we used Goals 3-7 with equal frequencies, the chance level of the "olfactory cues and possible goals" model could be set at $43.00 \%$, an average of the chance levels for Goals 3-7. 

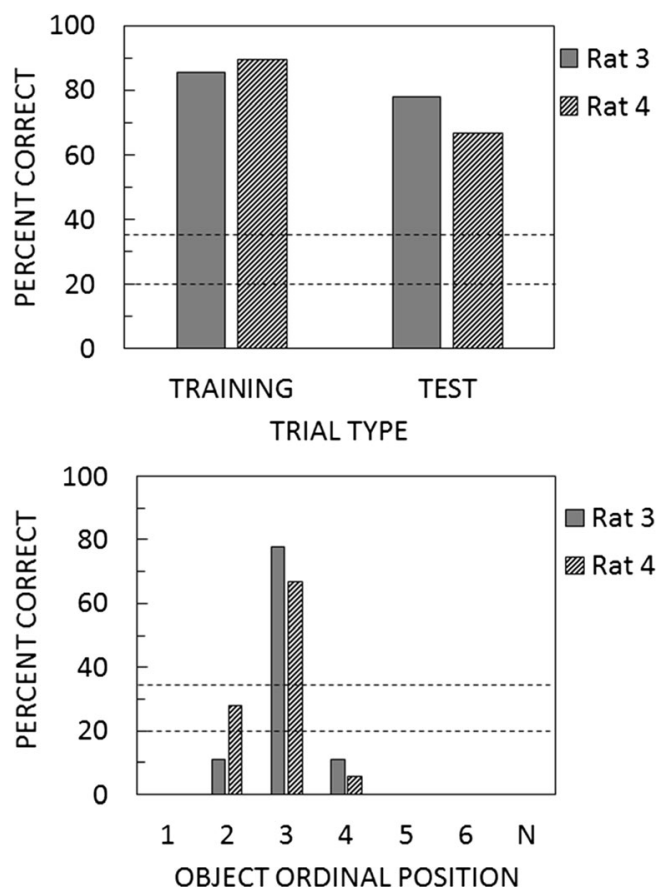

Fig. 4 Percentages of correct responses for the training and test objects (top panel) and percentages of responses to objects in each ordinal position in Experiment 1's probe test (bottom panel). "N" represents goal boxes with no object in front of them. The broken horizontal lines represent the different chance levels; details about the chance levels can be found in the text

Using this most conservative chance level of the "olfactory cues and possible goals" model to evaluate the significance of transfer performance to the test stimuli, the percentages of correct responses were significant for both rats (one-tailed binomial tests: Rat 3, $p=.003$; Rat $4, p=.037$ ).

The results of changing the total numbers of objects are shown in Fig. 5. The effect of the total number of objects was very slight, and performance was significantly better than the "objects and goals" model chance level of $35.93 \%$ in all conditions for both rats (one-tailed binomial test, $p \mathrm{~s}<.01$ ).

\section{Discussion}

Two out of four rats successfully learned numerical discrimination of the third object in an object row consisting of six objects. Since the spatial location of the objects was changed trial by trial, any specific spatial position of the target object or the correct goal box could not serve as an effective discriminative cue. Moreover, rats' performance was not affected by changing the total number of objects. The possibility of counting from the far right end of the row was also excluded, because the ordinal position of the target object from the right end varied depending on the total number of stimuli. This result also eliminated the possibility that rats used the relative position in a row of objects. For example, if the total number of objects was six, the correct third stimulus was always

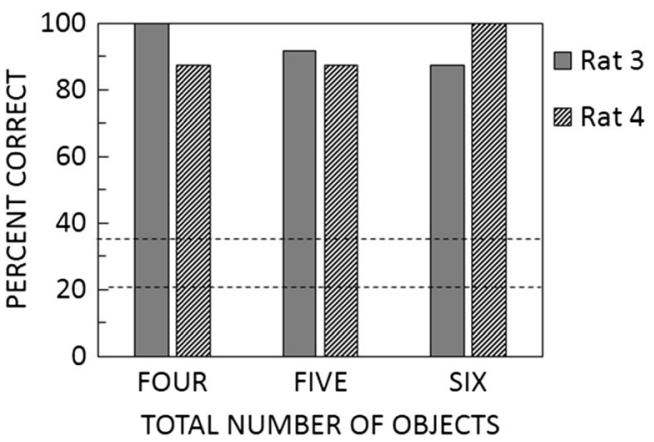

Fig. 5 Percentages of correct responses for conditions with varying numbers of objects in Experiment 1. Details about the different chance levels can be found in the text

located just to the left of the point dividing the row of six objects in half. However, the correct stimulus was placed just to the right of the halfway mark for a four-object row, or at the center of the five-object row. Therefore, the relative position of the correct stimulus in the object row could be eliminated from possible accounts for rats' successful acquisition of the task.

Two rats also showed reliable transfer of discrimination to novel object $\mathrm{D}$ after acquisition training with three different stimuli, A, B, and C. We reinforced responses to the second, third, and fourth stimuli in a row of test object D to control for olfactory cues and learning effects during the test trials. If rats responded to the smell of food pellets in the goal box, or if rats learned how to respond to the novel test object through reinforced experience during the test period, they should have responded equally to the second, third, and fourth objects. Given that the test performance of Rats 3 and 4 was reliably better than chance, an olfactory cue or reinforced experience could be eliminated as a reasonable explanation for the results of the probe test. Moreover, the transfer performance of Rats 3 and 4 was significantly higher than the most conservative "olfactory cues and possible goals" model of chance, $43.00 \%$, which might be expected if rats combined the conditions for possible correct goal boxes and olfactory cues from the food rewards or reinforcement experience during the test trials. Therefore, the results of Experiment 1 strongly suggest learning of abstract numerical discrimination in rats.

\section{Experiment 2}

In Experiment 1, two rats learned numerical discrimination of the third object in a row successfully. However, successful acquisition and presumed transfer of the third ordinal position was only confirmed with two subjects. Although the assignment of four different objects to the training and test stimuli was counterbalanced across subjects, it might have been possible that the test objects D for Rats 3 and 4 had, by chance, some specific similarity of physical features with the training 
stimuli, and that such a similarity contributed to their good transfer performance through a simple stimulus generalization process. Experiment 2 was designed to replicate Experiment 1 and confirm the further intersubject generality of transfer of the numerical discrimination to novel stimuli. If successful acquisition and transfer of discrimination to novel test stimuli were confirmed in additional subjects, the possibility that specific physical similarities mediated the transfer effect would decrease.

\section{Method}

Subjects The subjects were three experimentally naive, male Long-Evans rats, approximately 70 days old at the beginning of the experiment. Other aspects of the animals and their treatment were identical to those aspects of Experiment 1.

Apparatus The objects used in the initial training and probe tests were chosen from among metallic $150-\mathrm{ml}$ cans, glass bottles, ceramic dolls, metallic 350-ml cans, hemispherical capsules, artificial sunflowers, and transparent wine glasses. The assignment of these objects to items A, B, C, and D was counterbalanced among the subjects. Other aspects of the apparatus were identical to those of Experiment 1.

Procedure The procedure for pretraining was identical to that of Experiment 1, except that exploration of the apparatus was conducted for 4 days. After completion of pretraining, training was initiated. The procedure for this training was identical to that in Experiment 1, except for the following points. The learning criteria were $70 \%$ correct for two successive sessions and $50 \%$ correct for each training stimulus. During the probe test, 20 training trials with object $\mathrm{A}, \mathrm{B}$, or $\mathrm{C}$ and four probe test trials with novel object $\mathrm{D}$ were conducted in a daily 24-trial session. A probe test with D was inserted after every fifth training trial. Test sessions were conducted for four days (16 test trials in total).

Following the probe test, the rats were trained with objects $\mathrm{A}, \mathrm{B}, \mathrm{C}$, and D until they attained a learning criterion of $50 \%$ correct for each of the three objects and $70 \%$ correct, in total, for two successive sessions. Then they were tested with varying total numbers of objects A, B, C, or D-that is, four, five, or six objects-for 2 days.

\section{Results}

Figure 6 shows the percentages of correct responses during acquisition training. All three rats learned the acquisition task with three different stimuli, A, B, and C. Performance on the last two sessions of Phase 5 was significantly higher than the "objects and goals" model chance level of $35.93 \%$ (one-tailed binomial test, $p \mathrm{~s}<.01$ ).

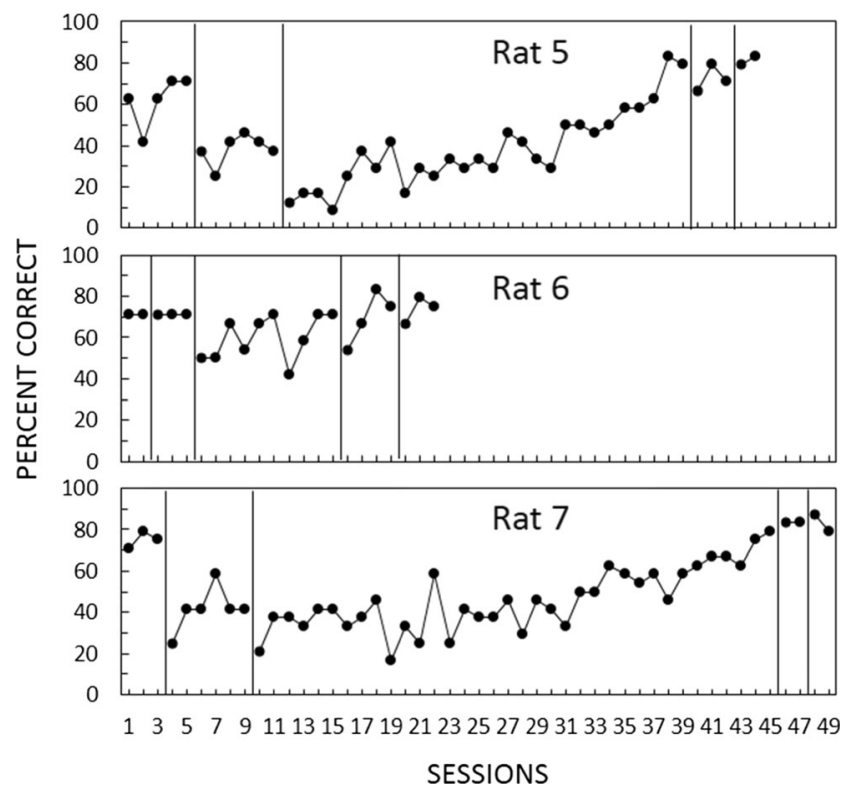

Fig. 6 Percentages of correct responses during acquisition training in Experiment 2. The solid vertical lines represent the beginning of each new training phase

Figure 7 shows the results of the probe test with novel stimulus D. Rats made most of their errors on the second and fourth objects, and very few errors were made to objects in more remote ordinal positions or to goals with no objects. Rats' performance was significantly better than chance for the
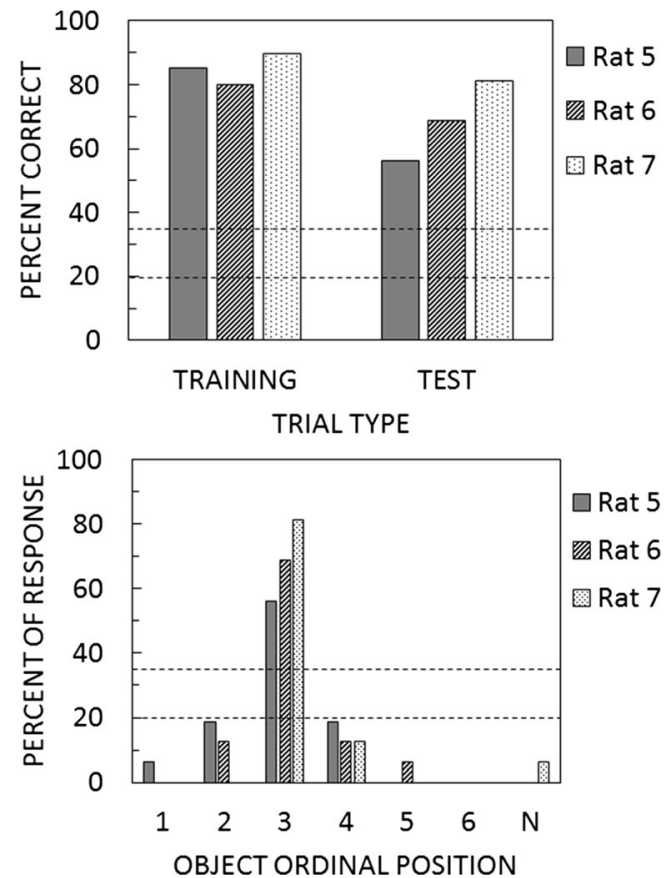

Fig. 7 Percentages of correct responses on training and test objects (top panel) and percentages of responses to objects in each ordinal position in Experiment 2's probe test (bottom panel). "N" represents goal boxes with no objects in front of them. The broken horizontal lines represent the different chance levels; details of these chance levels can be found in the text 
test trials (one-tailed binomial test, $p \mathrm{~s}<.01$ ), as well as for the training trials (one-tailed binomial test, $p \mathrm{~s}<.01$ ), even when compared to a chance level calculated from the combination of five possible boxes and the presence of objects ("objects and goals" model, $35.90 \%$ ). Compared to the most conservative chance level of $43.00 \%$, which was derived from a possible combination of olfactory cues from the food rewards and/or reinforced experience during test trials with the possible correct goal boxes ("olfactory cues and possible goals" model), performance on the test trials was not significant for Rat 5 (one-tailed binomial test, $p=.205$ ), but Rats 6 and 7 showed significant transfer performance compared with even the most conservative chance level (one-tailed binomial test: Rat 6, $p=.034$; Rat 7, $p=.002$ ).

The data from the transfer tests in Experiments 1 and 2 were also combined to compare training and test performance statistically. The combined mean percentages of correct response for the five rats were $86.12 \%$ and $70.14 \%$ for the training and test stimuli, respectively. A Trial Type $\times$ Subjects analysis of variance revealed a significant main effect of trial type $[F(1,4)=13.91, p=.020]$, showing significantly better performance for training than for test stimuli.

Figure 8 shows the results of a test with Rats $5-7$, in which the total number of objects varied among four, five, or six. The total number of objects in a row had almost no influence on the performance of each rat, and their performance was significantly better than the chance level of the "objects and goals" model $(35.93 \%)$ in all conditions (one-tailed binomial test, $p \mathrm{~s}<.01$ ). Again, the data from Experiment 1 (Rats 3 and 4) were combined with those from Experiment 2 to evaluate the effect of the total number of objects. The combined mean percentages of correct responses for the five rats were $90.00 \%, 88.33 \%$, and $90.00 \%$ for the four-, five-, and sixobject conditions, respectively. The main effect of number of objects $[F(2,8)=0.151, p=.867]$ was not significant in a Number of Objects $\times$ Subjects analysis of variance.

\section{Discussion}

All three rats in Experiment 2 successfully learned to discriminate the third object in an object row. Importantly, Rats 6 and 7 showed significant transfer performance compared with even the most conservative chance level of $43.00 \%$, derived if rats combined an olfactory cue of food reward and/or reinforced experience during the test trials with the possibly correct goal boxes. These results strongly suggest that possible nonnumerical explanations may be eliminated.

Successful acquisition and transfer of learning to novel test stimuli was shown for two rats in Experiment 1, and it was also replicated for the two rats in Experiment 2. Such replication helps to confirm the intersubject generality of numerical discrimination learning of objects and its transfer to novel stimuli in rats. Given that the assignment of objects to the

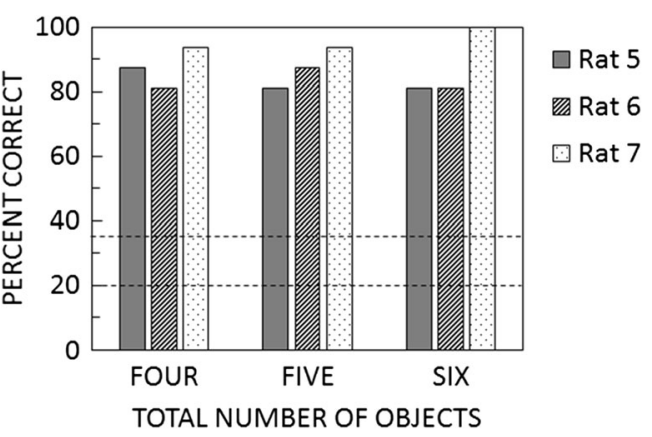

Fig. 8 Percentages of correct responses for conditions with varying numbers of objects in Experiment 2. Details about the different chance levels can be found in the text

training and test stimuli was counterbalanced across subjects, an explanation of the transfer effect in terms of any specific physical similarities shared between the training and test stimuli could be eliminated. Therefore, the transfer effect shown by these four rats strongly suggests that the rats learned an abstract numerical property during acquisition training and applied it to novel stimuli in the transfer test.

\section{Experiment 3}

A total of five out of seven rats successfully learned to respond to the third object in an object row in Experiments 1 and 2. Moreover, four of these five rats showed reliable transfer of learning to the novel test stimuli after training with three different training stimuli. However, a possible alternative explanation of the transfer that did not assume abstract numerical competence in rats was still available: That is, if rats ignored or were insensitive to differences among the training and test objects, good transfer performance to novel test stimuli would be expected. In this case, good transfer of learning would represent ignorance of the specific differences in physical features of the different objects used for training and testing, but it would not signify that a rat's abstract numerical discrimination could be applicable to dissimilar stimuli in common.

Experiment 3 was designed to elucidate this problem. The three rats from Experiment 2 continued to be trained with stimuli A, B, C, and D, as in Experiment 2. However, in Experiment 3, an odd stimulus was inserted among the five identical stimuli in a row, and rats were trained to respond to the third object in a row by ignoring the odd object. For example, in a row of AAABAA objects, in which the capital letters and their order represent the type and position of each object in the row, the third object A from the left was a positive stimulus, as in Experiment 2. By contrast, in a row of ABAAAA objects, the fourth object (i.e., the third A object) and not the third was a positive stimulus, because the odd object, B, was excluded from the count. Since the A, B, C, and $\mathrm{D}$ stimuli were all assigned to both the identical and the 
odd stimuli across trials, rats had not only to count objects on the basis of a numerical rule, but also to discriminate the objects from each other in order to exclude the odd stimulus from the count. Following acquisition training, the transfer of learning to novel test stimulus sets, consisting of novel objects $\mathrm{E}$ and $\mathrm{F}$, was examined. If rats could learn numerical discrimination of specific stimuli and could transfer that learning to test sets of novel stimuli, rats' numerical competence in learning an abstract ordering position would be strongly supported.

\section{Method}

Subjects The three rats from Experiment 2 were also used for Experiment 3, which began two days after Experiment 2 ended. During the interexperiment period, food deprivation was continued. The treatment of the animals was identical to that during Experiments 1 and 2.

Apparatus The assignment of objects, from the pool of seven types of items, to training stimuli A, B, C, and D for each rat, was identical to that of Experiment 2 and was counterbalanced across subjects. The two test objects, E and F, were selected from among the remaining three types of items, as listed in Experiment 2. Other aspects of the apparatus were identical to those of Experiments 1 and 2.

Procedure Rats were presented with stimulus sets consisting of five identical objects and an odd object in a trial (thus, six objects in total) and were required to respond to the third object from the left belonging to the set of identical objects, omitting the odd object from the count. The 12 stimulus sets were derived from the possible combinations of A, B, C, and D. These 12 sets were presented randomly in a 12-trial block and repeated twice daily for 24 trials per session. There were two types of trials, differing in whether the odd object was located prior to the fourth object or was the fourth, fifth, or sixth object in the row. In the former case, rats had to exclude the odd object from counting and respond to the fourth object in the row. By contrast, in the latter case, because the odd object was not included in the first three objects, rats did not have to exclude the odd object from counting and had to respond to the third object in the row. These two patterns of stimulus sets were presented randomly in every two-trial block. Therefore, the probabilities of whether or not rats had to exclude the odd object from counting on a trial were equally $50 \%$. The learning criterion for acquisition training was $70 \%$ correct for two successive sessions. On the same day that each rat attained the learning criterion, it was allowed to explore the new test stimuli. Four test objects, two each of $\mathrm{E}$ and F, were placed in a glass fish tank, and each rat was individually allowed to explore them freely for $20 \mathrm{~min}$.

After the completion of acquisition training, probe tests were conducted for six days. A total of 24 training trials and three probe trials were given in a daily session. Two novel probe sets, consisting of novel objects $\mathrm{E}$ and $\mathrm{F}$, were inserted as every ninth trial. Whether $\mathrm{E}$ or $\mathrm{F}$ was used as the identical or the odd stimulus was determined randomly for each two probe-trial blocks. For the test trials, the total number of objects varied between five and six, using four or five identical objects along with an odd object. For the probe sets, an odd stimulus was placed as the first, second, or third stimulus in an object row, and responses to the third and fourth objects were reinforced nondifferentially. That is, responses to the fourth object, based on selective counting, and the third object, based on nonselective counting of the stimuli, were equally reinforced with food rewards on the test trials. This enabled us to distinguish the transfer effects of selective counting on the probe test sets during the test trials from either olfactory cues from the food rewards or training effects.

\section{Results}

All three rats learned the acquisition task of selective counting very quickly. The number of sessions required to attain the learning criterion of the acquisition task was three for Rats 5 and 6, and four for Rat 7. Figure 9 shows the proportions of each response type-selective counting (responses to the fourth object, based on excluding the odd object from counting), nonselective counting (responses to the third object, based on including the odd object in counting), and other errors (responses to the other objects or goals with no objects) - during the 18 test trials. When the proportions of selective and nonselective counting were compared, so as to evaluate the learning transfer effect, the proportion of selective-counting responses was significantly higher than that of nonselective-counting responses for Rat 6 (one-tailed binomial test, $p=.006$ ) and Rat 7 (one-tailed binomial test, $p=$ .025 ), but not for Rat 5 (one-tailed binomial test, $p=.315$ ).

The proportions of selective-counting responses were analyzed separately for each total number of objects in a rowthat is, five or six. For Rats 6 and 7, the proportions of selective-counting responses were relatively high in both the five-object condition $(6 / 9=66.7 \%$ and $7 / 9=77.8 \%$, respectively) and the six-object condition $(6 / 9=66.7 \%$ and $6 / 9=$ $66.7 \%$, respectively). In contrast, for Rat 5 the proportion of selective-counting performance was poor in the five-object condition $(3 / 9=33.33 \%)$, although it was good in the sixobject condition $(7 / 9=77.8 \%)$, resulting in overall nonsignificant selective-counting performance.

\section{Discussion}

All three rats learned the task of selectively counting identical stimuli within four sessions. Considering that 22-49 sessions were needed for acquisition of the original counting task in Experiment 2, the selective-counting task in Experiment 3 was 
궁 OTHER ERROR

\section{NON-SELECTIVE COUNTING}

$\square$ SELECTIVE COUNTING

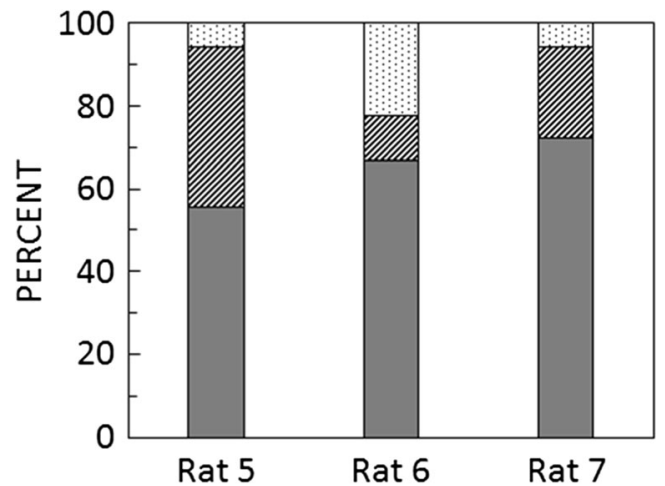

Fig. 9 Percentages of each rat's response type in Experiment 3's probe test

learned very quickly. These results suggest that rats had a tendency to exclude the odd stimulus from counting at the initial stage of selective-counting training, rather than learning to exclude it by trial and error through the acquisition training of Experiment 3. Even when rats were trained with the acquisition task in Experiment 2, in which only identical objects were used, extraneous stimuli were around the target objects - for example, goal doors with no object in front of them. Rats might have learned to exclude such extraneous stimuli from counting during Experiment 2, and this tendency to exclude extraneous stimuli might have transferred to the selective-counting task in Experiment 3. Additionally, it is known that rats discriminate an odd stimulus among identical stimuli spontaneously, without explicit oddity discrimination training (Forwood, Bartko, Saksida, \& Bussey, 2007). Therefore, an odd stimulus in the present setting might also be naturally salient and be processed differently from the identical stimuli. An interesting experiment would be to investigate whether rats can be trained explicitly to switch between excluding and including an odd stimulus in counting, by explicit stimulus control training.

Rats 6 and 7 showed reliable transfer of selective counting to novel test stimuli. As we described in the introduction to Experiment 3, transfer of learning in a task consisting of only identical stimuli, as in Experiments 1 and 2, could be explained by mere ignorance or insensitivity to a specific difference existing among the training and test objects. But the task of selectively counting identical stimuli in Experiment 3 required rats to discriminate various objects from each other. Therefore, successful acquisition of the selective-counting task and its transfer to novel stimuli by the two rats strongly suggests that rats can learn the abstract ordering position of objects.

Although Rat 5 acquired the selective-counting task, it only performed well in the six-object condition, not in the five- object condition of the transfer test. Since acquisition training of selective counting was given only in a six-object condition in Experiment 3, counting the identical stimuli from either the left or the right end could have been an effective strategy. That is, the third identical object from both the left and right ends was always the correct stimulus, although both of these response strategies would require strict discrimination of the identical stimuli from the odd one. The results of the test of the total number of the objects in Experiment 2 suggested that Rat 5 responded to the target object by counting from the left end in the object row. We cannot find any plausible reason for such a possible shift in response strategy, but it could reasonably explain the good performance in six-object trials $(77.8 \%)$, but not in five-object trials $(33.3 \%)$, because counting from the right end would produce erroneous results only in the five-object trials. That acquisition training was only conducted in the six-object condition might have affected Rat 5's performance. Obviously, reexamination of training by varying the total number of objects in a row will be needed to show clearer evidence of abstract selective counting of specific stimuli in rats.

\section{Experiment 4}

In Experiment 3, rats could successfully learn to respond to the third object, excluding an odd object in a row. Experiment 4 was conducted to examine whether rats could be insensitive to or ignore physical differences among the objects. The rats from Experiment 3 continued to be trained with two kinds of trials: object rows consisting of six identical objects on half of the trials, and six different objects on the remaining trials. If rats could switch their response strategies between ignoring and not ignoring differences in objects, they would learn to respond correctly in both conditions.

\section{Method}

Subjects The three rats from Experiment 3 continued as subjects. Experiment 4 began three days after Experiment 3 ended. During the interexperiment period, food deprivation was continued. Treatment of the animals was identical to that in Experiments 1-3.

Apparatus The assignment of objects to training stimuli A, B, $\mathrm{C}, \mathrm{D}, \mathrm{E}$, and $\mathrm{F}$ for each rat was identical to that in Experiment 3. Other aspects of the apparatus were identical to those of Experiments 1-3.

Procedure Rats were trained to respond to the third object in a six-object row. There were two types of trials. For half of the trials, the object rows consisted of six identical objects: AAAAAA, BBBBBB, CCCCCC, DDDDDD, EEEEEE, or 
FFFFFF. For the remaining trials, the object rows consisted of different arrangements of $\mathrm{A}, \mathrm{B}, \mathrm{C}, \mathrm{D}, \mathrm{E}$, and $\mathrm{F}$ (CABFED, FDCBEA, etc.). These two types of trials were presented randomly in two-trial blocks in a daily session of 24 trials. Training was conducted for two sessions. Other aspects of the procedure were identical to the acquisition training in Experiments 1 and 2 .

\section{Results}

Figure 10 shows performance for the identical-objects and different-objects trials separately. Performance did not differ markedly between trial types and reached a high level on the second session for both conditions. A Trial Type $\times$ Session $\times$ Subjects analysis of variance showed no reliable main effects of trial type $[F(1,2)=0.00]$ and session $[F(1,2)=1.73, p=$ $.319]$, or an interaction of trial type and session $[F(1,2)=$ $0.00]$.

\section{Discussion}

Experiment 3 suggested that rats could learn to respond to specific identical stimuli and exclude an odd stimulus mixed into an object row. In Experiment 4, the same rats from Experiment 3 quickly learned to respond to the third stimulus in a row of different objects as well as in an identical-objects row. These results suggest that rats can switch the stimulus property to be counted, depending on the task demands.

A similar phenomenon is known as categorical flexibility in humans. For example, we can count two apples and three oranges separately, but also in combination as "five pieces of fruit." In runway experiments, it has been reported that rats showed categorical flexibility to a certain extent. For example, Burns, Goettl, and Burt (1995) showed that rats trained with R'RRN series showed positive transfer of learning to both the RRN and the RRRN series, as compared with appropriate control conditions ( $\mathrm{R}, \mathrm{R}$ ', and $\mathrm{N}$ refer to qualitatively different food rewards and nonrewards, respectively). These results suggest that rats can count two different rewards, R and R', not only separately, but also in combination. In the present study, the same rats that learned to respond only to identical objects by excluding an odd stimulus, in Experiment 3, also learned very quickly to respond to different objects nonspecifically. These results suggest that categorical flexibility in rats may also be demonstrated in an object-counting task.

However, the reason why rats could switch their strategies so quickly from excluding (Exp. 3) to including (Exp. 4) different stimuli in counting is still unclear. Specifically, Rat 7 showed asymptotic performance from the first session. There were no explicit cues that informed the shift from the selective-counting task in Experiment 3 to the task of counting different objects in Experiment 4. One possible account might be that rats have a spontaneous tendency to exclude different
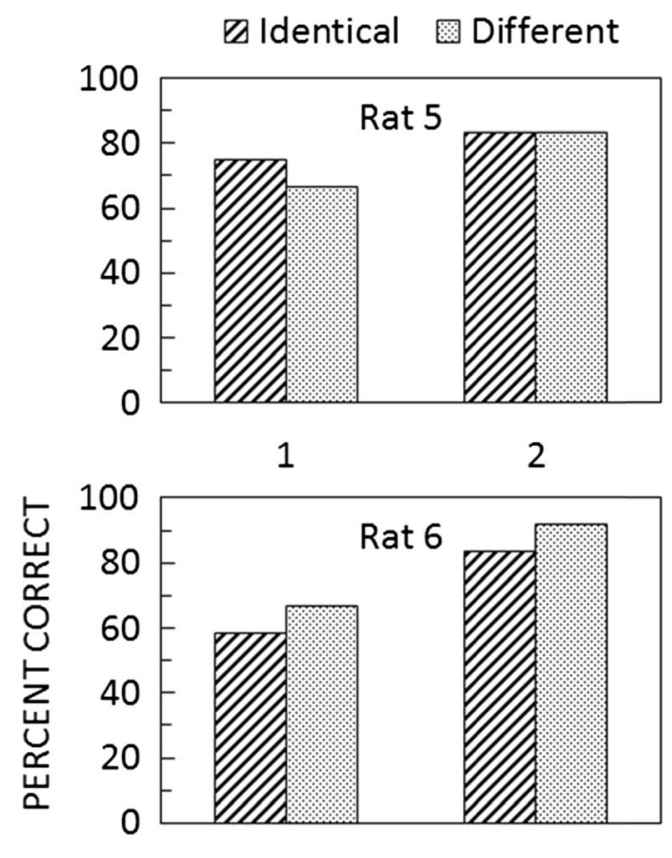

12

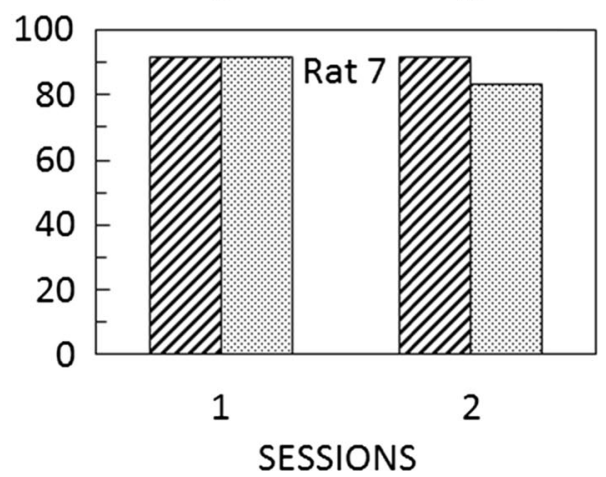

Fig. 10 Percentages of correct responses by each rat in the identicalobject and different-object conditions. The object rows consisted of six identical objects in the identical-object condition, and six different objects in the different-object condition

stimuli from counting when a very small number of different stimuli (e.g., one in Exp. 3) are mixed in with a large number of identical stimuli, because the different stimuli provide perceptual oddity (Forwood et al., 2007) and are processed differently from identical stimuli. Rats may include different stimuli in counting spontaneously when there are no identical stimuli, as in Experiment 4, because the different stimuli do not offer perceptual oddity, given the of lack of identical stimuli. Obviously, additional examination of these possibilities, by manipulating the numbers of identical and different stimuli in a row, will be needed in addition to an examination of explicit training in stimulus control of shifts in learning strategies, including or excluding different stimuli in counting, as we mentioned in the Discussion of Experiment 3. A promising future study with rats would be an examination of explicit training of categorical flexibility in the object-counting task. 


\section{General discussion}

Five out of seven rats learned to respond to the third object in an object row. In this discrimination task, the spatial position of a target stimulus could not act as an effective discriminative cue, because it was changed trial by trial. The relative position of a target stimulus in an object row also could not serve an effective discriminative cue, because a change in the total number of objects in a row did not affect the rats' performance. More importantly, four out of the five rats also attained significant performance when their individual data were compared to the strictest chance level of $43.00 \%$. These results strongly suggest that rats can learn the abstract ordering position of a target stimulus and that possible nonnumerical artifacts can be controlled.

Unlike Kamijo and Taniuchi (2015), who reported a failure to transfer learning to novel test stimuli despite successful acquisition of discrimination of the third object in a row, a significant transfer of discrimination was observed in the present study. There were two major methodological differences between the present study and Kamijo and Taniuchi's study. The first was the inclusion of a habituation treatment for the test stimuli. Kamijo and Taniuchi presented their test objects as completely novel stimuli, whereas in this study, we allowed rats to explore the test stimuli in advance of testing. Kamijo and Taniuchi also reported that rats responded to goal boxes with no object in front more frequently on test than on training trials. This result might possibly reflect stronger neophobia for the novel test stimuli (e.g., Wallace \& Barnett, 1990) than for the familiar training stimuli, and this possible neophobia might have interfered with the test performance in Kamijo and Taniuchi (2015). Although we cannot evaluate the possible effects of habituation treatment for the test stimuli, because the present study included no relevant control condition, considering that the mean percentages of responses to goals with no object on test trials were $1.25 \%$ in the present study and $17.83 \%$, on average, in Kamijo and Taniuchi (2015), familiarization of the test stimuli by habituation treatment might have contributed to a significant transfer effect by suppressing a possible exploration tendency or any neophobia toward the test stimuli.

A second major difference between Kamijo and Taniuchi (2015) and the present study was control of the odor of the food rewards. Kamijo and Taniuchi placed food rewards in all of the goal boxes, in order to control for any possible olfactory cue from those rewards, and locked the small one-way door, blocking access to the food cup, in the incorrect goal boxes to prevent rats from getting those rewards. Although this procedure was a strict means of control for any possible odor cue from the food rewards, the researchers observed that rats sometimes persisted in trying to open the small one-way door in the incorrect goal boxes (Kamijo \& Taniuchi, 2015). When rats encounter correct or incorrect feedback in terms of reinforcement outcomes, they need to associate the reinforcement outcome with their immediate discriminative response in order to learn the task. In this situation, the persistent emission of irrelevant responses in an incorrect goal box possibly interfered with forming an association between the reinforcement outcomes and discriminative responses. Considering this possible interferential effect, we placed the food reward only in the correct goal box during training the trials in this study. The rats encountered empty food cups in incorrect goal boxes, and any persistent irrelevant behavior related to trying to get food rewards was not observed. Although we cannot compare the acquisition speeds in these two studies directly, because of the several differences in experimental procedure, it took about 2, 000-3,000 trials in Kamijo and Taniuchi's study, versus about $500-1,200$ trials in the present study, to acquire the discrimination task with three different objects. Suppressing persistent irrelevant behaviors by not setting food rewards in incorrect goal boxes might have contributed to the faster learning of the task in the present study.

One may be concerned that an odor cue from food rewards might have guided rats' discriminative responses because a food reward was only placed in the correct goal box during training. However, four rats showed significant performance at even the most conservative chance level, which took a possible odor cue into account when evaluating test performance. That is, if rats could combine the information about the possible goal boxes with an odor cue from food rewards placed in the goal boxes behind the second, third, and fourth test objects on the test trial, the strictest chance level would be $43.00 \%$. Four out of the five rats who mastered acquisition training with objects $\mathrm{A}, \mathrm{B}$, and $\mathrm{C}$ showed significant performance. Although we cannot completely exclude the possible effects of an olfactory cue from the food reward on rats' performance, these statistical results show that an odor cue from a food reward alone cannot adequately explain the transfer performance.

Another possible explanation might be that rats searched for the general location of food rewards on the basis of an odor cue. In that case, they might have responded more strongly to middle of three food rewards on the test trials because it possibly received indirect odors from both the first (behind the second object) and third (behind the fourth object) food rewards, as well as the direct odor of the second food reward (behind the third object). The second object must have received a relatively weaker indirect odor cue than the third object, because it could only receive indirect odors from one proximate food reward (behind the third object) and one distant food reward (behind the fourth object), and the same might have been true for the fourth object. However, this view cannot explain the significant transfer of selective-counting performance in Experiment 3. On the test trials of the selective-counting task, food rewards were always set behind both the third and fourth objects, regardless of the position of an odd object. In this situation, the possible strengths of direct 
and indirect odor cues must have been equal for the third and fourth objects. Therefore, a general search for a food reward, based on direct and indirect odor cues, cannot explain the significant selective-counting transfer performance in Experiment 3. However, reexamination with a stricter control of possible odor cues by means of a test with an extinction procedure might be needed to rule out the influence of odor cues on rats' performance.

Therefore, we conclude that the rats in the present study did learn abstract ordering positions applicable to various objects. Experiment 3 showed that rats could learn to count only the specific identical stimuli, excluding the odd stimulus added to an object row. Moreover, two out of three rats showed significant transfer of the selective-counting behavior to test sets consisting of novel stimuli. As we mentioned earlier, transfer of learning of a non-selective-counting task, as in Experiments 1 and 2, can be explained by simple insensitivity to or ignorance of the differences among various objects. However, the selective-counting task in Experiment 3 could never be learned without a discrimination between the identical stimuli and an odd stimulus. Therefore, the reliable learning and transfer of the selective-counting behavior in Experiment 3 strongly suggests rats' capacity to learn an abstract numerical property of objects.

Similar selective counting has been reported in runway experiments. For example, Capaldi and Miller (1988) showed that rats could learn to anticipate a final nonreward trial in four different reward series of RRN, R'R'N, R'RRN, and RR'R N concurrently (R, $R^{\prime}$, and $N$ here refer to qualitatively different food rewards and nonreward, respectively). To identify the final nonreward in the series, rats had to count just two identical reward events, ignoring any odd reward. Moreover, as we mentioned earlier, Burns, Goettl, and Burt (1995) showed that rats trained with a R'RRN series showed positive transfer of learning to both RRN and RRRN series, as compared with appropriate control conditions. Their results suggest that rats can count R and R' not only separately, but also in combination. In the present study, the same rats that learned to count only identical objects in Experiment 3 also learned very quickly to count different objects nonspecifically in Experiment 4. These results strongly suggest that categorical flexibility in counting might be found in an object-counting task, as well as in runway experiments. It would be interesting to examine explicit training of categorical flexibility in object-counting tasks in rats.

As we explained in Experiment 2, although rats' performance to the test stimuli was reliably better than chance in Experiments 1 and 2, it was also reliably poorer than to the training stimuli. Katz and Wright (2006) described different levels of transfer to novel stimuli in terms of full or partial transfer. It is said that partial transfer represents partial concept learning; that is, discriminative behavior is controlled not only by the abstract concept, but also by certain stimulus-specific cues that involve learning specific physical features of the training stimuli. Therefore, although the reliable transfer to novel test stimuli in the present study ensures that rats learned, at least partially, a type of abstract numerical concept, it should be possible to enhance numerical concept learning in rats. In relational same-different concept learning studies, we know that increasing the number of exemplars used in acquisition training leads to better transfer performance, perhaps because animals abandon stimulus-specific learning given a large number of stimuli (Katz \& Wright, 2006; Wright \& Katz, 2006). We might expect similar effects in numerical concept learning in rats. In this study, we trained rats with just three or four kinds of training stimuli before the transfer test. To explore additional possibilities of abstract numerical concept learning in rats, it would be worthwhile to examine numerical discrimination learning of objects with a greater variety of training stimuli.

In this series of experiments, we examined rats' ability to identify the third object in a row. A simple question might arise: How many objects can a rat enumerate? This matter is related to the important topic of subitizing, which is the rapid and effortless perceptual apprehension of a small number of items, generally up to four, through preattentive mechanisms (Kaufman, Lord, Reese, \& Volkmann, 1949; Neider, 2005). In contrast, counting refers to a slow, effortful mental enumeration process applied to relatively larger numbers of items, generally greater than four in human adults (Oyama, Kikuchi, \& Ichihara, 1981). Because the experimental setup of our study might have allowed rats to perceive multiple objects simultaneously, and since the target aggregation, three objects, was less than four, rats might have responded to the target object not only by counting, but also by subitizing the target aggregation. Therefore, additional assessments will be needed to distinguish a true counting process from subitizing. This goal could be accomplished in at least two ways. First, we could examine the acquisition of discrimination learning of a larger number of item stimuli. Since subitizing can be applied to only a relatively small number of item stimuli, generally four or less, if rats could learn a numerical task in which the discrimination of more than four stimulus items was required, true counting, rather than subitizing, would be the most likely form of processing. Second, since subitizing is mainly used with stimuli presented simultaneously or presented sequentially within very short intervals (Beran \& Beran, 2004), if rats could learn to count objects presented serially with sufficiently long intervals, this would be strongly suggestive of counting rather than subitizing. We might be able to accomplish this goal by modifying the apparatus to ensure that the rats encounter each object serially-for example, by separating the row of objects with one-way doors.

Examining the discrimination of a larger number of objects will also be important for exploring the influence of another counting process. It has been reported that nonhuman animals, 
as well as humans, estimate the set size of stimuli on the basis of the analog magnitude or approximate number system (see, e.g., Neider, 2005). Unlike subitizing, there is no upper limit of the number of stimuli the analog magnitude system can estimate, but the estimation becomes less precise as the number of stimuli increases. For example, when rats were trained to make some specific number of responses to targets, the variance of the number of responses made by the rats increased as the target number increased (Mechner, 1958; Platt \& Johnson, 1971). It has also been reported that rats (Meck \& Church, 1983) and monkeys (Jordan \& Brannon, 2006) judge the similarity between two and four items to be as similar as that between four and eight items. Thus, it is known that estimation by the analog magnitude system follows Weber's law - that is, the ratio between numerosities is critical for discriminability. If the analog magnitude system was responsible for the rats' numerical discriminations in the present experiments, we would predict that errors to incorrect stimuli would increase in proportion to the magnitude of the ordinal position of the target stimulus in a row.

It would be constructive to examine discrimination of a larger number of objects in a serial presentation apparatus in order to more fully ascertain the extent of abstract numerical discrimination learning in rats. Additionally, in the present study, the absolute ordinal position of the target stimulus was always third. Training identical rats to respond to different target ordinal positions, depending on different conditional cues, would be an interesting means to add to our knowledge of the flexibility of numerical competence in rats.

Primate studies have revealed that numerical cues are salient for rhesus monkeys, because they use numerical cues even when other stimulus dimensions are also available (Cantlon \& Brannon, 2007). Rhesus monkeys also showed cross-modal matching of the number of sounds and the number of visual stimuli, suggesting that their number representations, like those of humans, are not fettered to a specific stimulus modality (Jordan, MacLean, \& Brannon, 2008). Rhesus monkeys (Brannon \& Terrace, 1998, 2000) and capuchin monkeys (Judge, Evans, \& Vyas, 2005) responded to the ordinal relationship of a number of different figures on a computer monitor correctly. A chimpanzee responded correctly to the number of visual stimuli by using arbitrary symbols (Matsuzawa, 1985). Chimpanzees could estimate the total number of food items shown to them sequentially, and they also could estimate the remainder when one food item was subtracted (Beran, 2004). None of these abilities - involving the salience of numerical cues, the cross-modal abstractness of numerical representations, ordinal judgment, symbolic matching of the number of items and arbitrary symbols, and addition or subtraction - have yet been demonstrated in rodents. In this study, we succeeded in demonstrating rats' ability to count objects in a flexible and somewhat abstract way. These findings also suggest promising new experiments through which to probe rats' responses to greater complexity in numerical processing.

Author note This article is based on experiments conducted by the second and third authors for their graduate theses at Kanazawa University, under the supervision of the first author. This work was partly supported by JSPS KAKENHI Grant Number 24530913.

\section{References}

Beran, M. J. (2004). Chimpanzees (Pan troglodytes) respond to nonvisible sets after one-by-one addition and removal of items. Journal of Comparative Psychology, 118, 25-36. doi:10.1037/ 0735-7036.118.1.25

Beran, M. J., \& Beran, M. M. (2004). Chimpanzees remember the results of one-by-one addition of food items to sets over extended time periods. Psychological Science, 15, 94-99. doi:10.1111/j.09637214.2004.01502004.x

Brannon, E. M., \& Roitman, J. D. (2003). Nonverbal representations of time and number in animals and human infants. In W. H. Meck (Ed.), Functional and neural mechanisms of interval timing: Methods and new frontiers in neuroscience (pp. 143-182). Boca Raton: CRC Press. doi:10.1201/9780203009574

Brannon, E. M., \& Terrace, H. S. (1998). Ordering of the numerosities 1 to 9 by monkeys. Science, 282, 746-749.

Brannon, E. M., \& Terrace, H. S. (2000). Representation of the numerosities 1-9 by rhesus macaques (Macaca mulatta). Journal of Experimental Psychology: Animal Behavior Processes, 26, 3149. doi:10.1037/0097-7403.26.1.31

Breukelaar, J. W. C., \& Dalrymple-Alford, J. C. (1998). Timing ability and numerical competence in rats. Journal of Experimental Psychology: Animal Behavior Processes, 24, 84-97. doi:10.1037/ 0097-7403.24.1.84

Burns, R. A., Goettl, M. E., \& Burt, S. T. (1995). Numerical discrimination with arrhythmic serial presentations. Psychological Record, 45, 95-104.

Cantlon, J. F., \& Brannon, E. M. (2007). How much does number matter to a monkey (Macaca mulatta)? Journal of Experimental Psychology: Animal Behavior Processes, 33, 32-41. doi:10.1037/ 0097-7403.33.1.32

Capaldi, E. J., \& Miller, D. J. (1988). Counting in rats: Its functional significance and the independent cognitive processes that constitute it. Journal of Experimental Psychology: Animal Behavior Processes, 14, 3-17. doi:10.1037/0097-7403.14.1.3

Castro, L., Lazareva, O. F., Vecera, S. P., \& Wasserman, E. A. (2010). Changes in area affect figure-ground assignment in pigeons. Vision Research, 50, 497-508. doi:10.1016/j.visres.2009.12.016

D’Amato, M. R., Salmon, D. P., \& Colombo, M. (1985). Extent and limits of the matching concept in monkeys (Cebus apella). Journal of Experimental Psychology: Animal Behavior Processes, 11, 35-51. doi:10.1037/0097-7403.11.1.35

Davis, H., \& Albert, M. (1986). Numerical discrimination by rats using sequential auditory stimuli. Animal Learning \& Behavior, 14, 5759.

Davis, H., \& Albert, M. (1987). Failure to transfer or train a numerical discrimination using sequential visual stimuli in rats. Bulletin of the Psychonomic Society, 25, 472-474.

Davis, H., \& Bradford, S. A. (1986). Counting behavior by rats in a simulated natural environment. Ethology, 73, 265-280.

Davis, H., MacKenzie, K. A., \& Morrison, S. (1989). Numerical discrimination by rats (Rattus norvegicus) using body and vibrissal touch. Journal of Comparative Psychology, 103, 45-53. doi:10.1037/ 0735-7036.103.1.45 
Ennaceur, A., Michalikova, S., \& Chazot, P. L. (2009). Do rats really express neophobia towards novel objects? Experimental evidence from exposure to novelty and to an object recognition task in an open space and an enclosed space. Behavioural Brain Research, 197, 417-434. doi:10.1016/j.bbr.2008.10.007

Forwood, S. E., Bartko, S. J., Saksida, L. M., \& Bussey, T. J. (2007). Rats spontaneously discriminate purely visual, two-dimensional stimuli in tests of recognition memory and perceptual oddity. Behavioral Neuroscience, 121, 1032-1042. doi:10.1037/0735-7044.121.5. 1032

Gelman, R., \& Gallistel, C. R. (1978). The child's understanding of number. Cambridge: Harvard University Press.

Jordan, K. E., \& Brannon, E. M. (2006). A common representational system governed by Weber's law: Nonverbal numerical similarity judgments in 6-year-olds and rhesus macaques. Journal of Experimental Child Psychology, 95, 215-229. doi:10.1016/j.jecp. 2006.05.004

Jordan, K. E., MacLean, E. L., \& Brannon, E. M. (2008). Monkeys match and tally quantities across senses. Cognition, 108, 617-625. doi:10. 1016/j.cognition.2008.05.006

Judge, P. G., Evans, T. A., \& Vyas, D. K. (2005). Ordinal representation of numeric quantities by brown capuchin monkeys (Cebus apella). Journal of Experimental Psychology: Animal Behavior Processes, 31, 79-94. doi:10.1037/0097-7403.31.1.79

Kamijo, M., \& Taniuchi, T. (2015). Acquisition and limited transfer of numerical discrimination of object stimuli in rats. Psychological Record, 65, 289-300. doi:10.1007/s40732-014-0105-0

Katz, J. S., \& Wright, A. A. (2006). Same/different abstract-concept learning by pigeons. Journal of Experimental Psychology: Animal Behavior Processes, 32, 80-86. doi:10.1037/0097-7403.32.1.80

Kaufman, E. L., Lord, M. W., Reese, T. W., \& Volkmann, J. (1949). The discrimination of visual number. American Journal of Psychology, 62, 498-525. doi:10.2307/1418556

Matsuzawa, T. (1985). Use of numbers by a chimpanzee. Nature, 315 , $57-59$.

Mechner, F. (1958). Probability relations within response sequences under ratio reinforcement. Journal of the Experimental Analysis of Behavior, 1, 109-121.

Meck, W. H., \& Church, R. M. (1983). A mode control model of counting and timing processes. Journal of Experimental Psychology: Animal Behavior Processes, 9, 320-334. doi:10.1037/0097-7403.9.3.320

Miletto Petrazzini, M. E., Agrillo, C., Izard, V., \& Bisazza, A. (2015). Relative versus absolute numerical representation in fish: Can guppies represent "fourness"? Animal Cognition, 18, 1007-1017. doi:10.1007/s10071-015-0868-y

Neider, A. (2005). Counting on neurons: The neurobiology of numerical competence. Nature Reviews, 6, 177-190. doi:10.1038/nrn1626

Oyama, T., Kikuchi, T., \& Ichihara, S. (1981). Span of attention, backward masking, and reaction time. Perception \& Psychophysics, 29, 106-112.

Pepperberg, I. M. (1994). Numerical competence in an African gray parrot (Psittacus erithacus). Journal of Comparative Psychology, 108, 36-44. doi:10.1037/0735-7036.108.1.36

Pepperberg, I. M. (2012). Further evidence for addition and numerical competence by a Grey parrot (Psittacus erithacus). Animal Cognition, 15, 711-717.

Perdue, B. M., Talbot, C. F., Stone, A. M., \& Beran, M. J. (2012). Putting the elephant back in the herd: Elephant relative quantity judgments match those of other species. Animal Cognition, 15, 955-961. doi: 10.1007/s10071-012-0521-y

Platt, J. R., \& Johnson, D. M. (1971). Localization of position within a homogeneous behavior chain: Effects of error contingencies. Learning and Motivation, 2, 386-414.

Rugani, R., Regolin, L., \& Vallortigara, G. (2008). Discrimination of small numerosities in young chicks. Journal of Experimental Psychology: Animal Behavior Processes, 34, 388-399. doi:10. 1037/0097-7403.34.3.388

Suzuki, K., \& Kobayashi, T. (2000). Numerical competence in rats (Rattus norvegicus): Davis and Bradford (1986) extended. Journal of Comparative Psychology, 114, 73-85. doi:10.1037/0735-7036. 114.1.73

Wallace, R. J., \& Barnett, S. A. (1990). Avoidance of new objects by the black rat (Rattus rattus) in relation to object presentation and object change. International Journal of Comparative Psychology, 3, 253 265.

Wright, A. A., \& Katz, J. S. (2006). Mechanisms of same/different concept learning in primates and avians. Behavioural Processes, 72, 234-254.

Xia, L., Emmerton, J., Siemann, M., \& Delius, J. D. (2001). Pigeons (Columba livia) learn to link numerosities with symbols. Journal of Comparative Psychology, 115, 83-91. doi:10.1037/0735-7036. 115.1.83

Xia, L., Siemann, M., \& Delius, J. D. (2000). Matching of numerical symbols with number of responses by pigeons. Animal Cognition, 3, 35-43. 